\title{
CLIL implemented and scaffolded in an EFL higher education context: Engineering research methodology course
}

\author{
Budsaba Kanoksilapatham ${ }^{1 *}$ and Attapol Khamkhien \\ ${ }^{1}$ Faculty of Management Science, Silpakorn University, 1 Moo 3, Sam Phraya, \\ Cha-am, Phetchaburi, Thailand 76120 \\ ${ }^{2}$ Language Institute, Thammasat University, 99 Moo 18, Paholyothin Road, \\ Klong Nueng, Klong Luang, Pathumthani, Thailand 12120
}

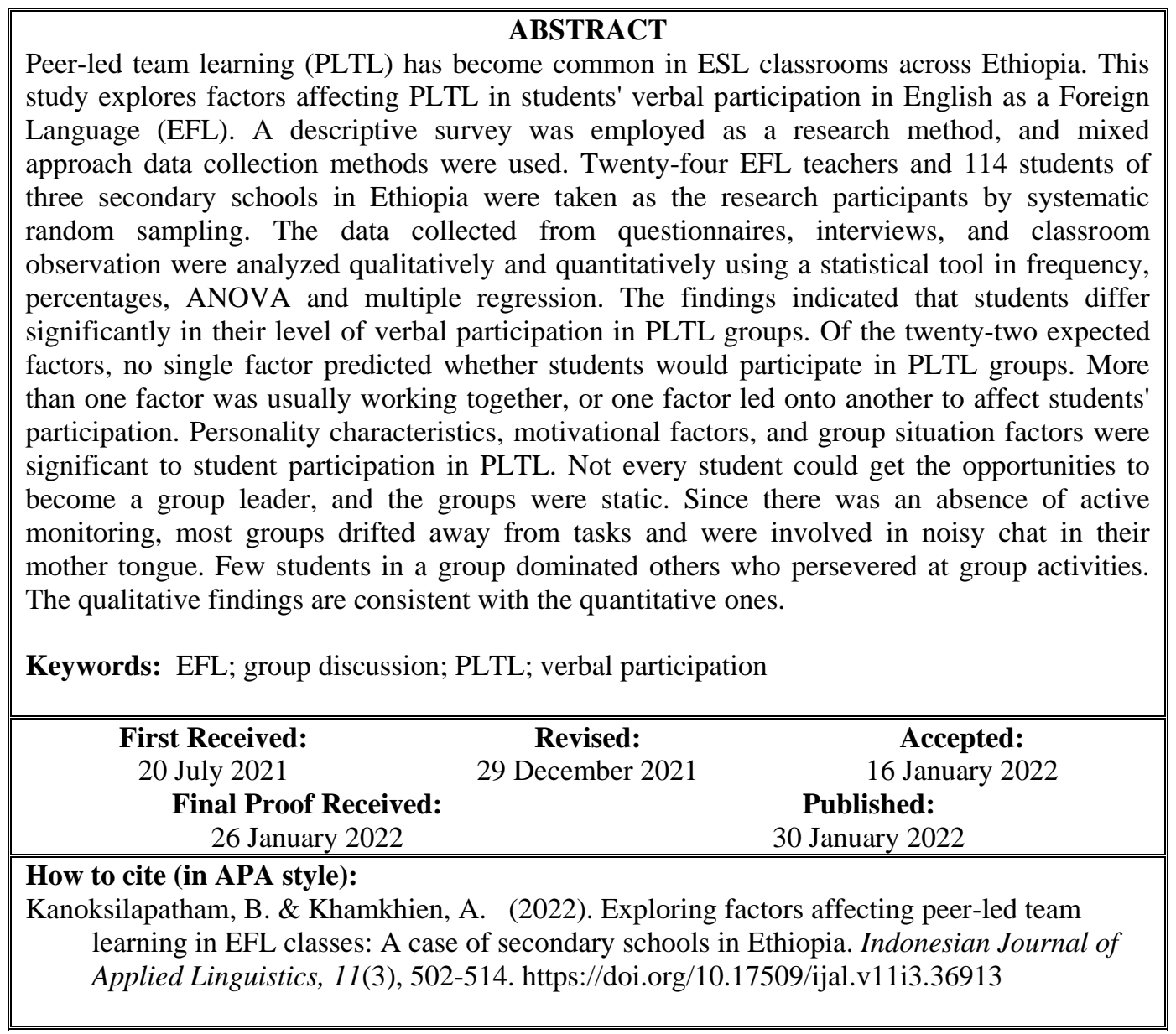

\section{INTRODUCTION}

The increasing global and local demands to improve English proficiency have augmented the significance of English education across the entire paradigm. In Thailand, improving Thai students' English has been a burgeoning challenge, attested by unsatisfactory national and international English exam scores despite a large funding commitment to the Ministry of Education. This grim outlook of Thai learners' English has been pervasive, across the entire paradigm of English education (Kanoksilapatham, 2020). Meanwhile, Content Language Integrated Learning )CLIL( was created by Marsh in 1994. The principal tenet of CLIL, as nicely summed up by Coyle et al. )2010) is that CLIL "a dual-focused educational approach in which an additional language is used for the learning and teaching of both content and language." This implies that, according to CLIL principles, a subject teacher must also learn the

\footnotetext{
* Corresponding Author

Email: kanoksib@hotmail.com
} 
additional language to be used as the medium of instruction when teaching the subject. Similarly, language teachers also need to learn more about the subject content if CLIL is to be successfully adopted. Given the dismal scenario of English education in Thailand and the potential pedagogical usefulness of CLIL, a scrutiny of CLIL and its implementation is warranted.

CLIL has been adopted worldwide as a successful teaching approach that promotes more motivated and sustained learning attainment )Marsh, 2000). A wealth of studies adopting CLIL principles have been conducted )e.g., Fan \& Lo, 2016; Fazzi \& Lasagabaster, 2021; Kalay, 2021; Navarro-Guzman et al., 2021; Mahan, 2020; Pérez Cañado, 2020; Thuy \& Nguyen, 2016; Yang, 2015). These previous CLIL empirical studies have congruently demonstrated its positive impacts on encouraging students to concurrently learn the content subjects and the target language across disciplines (e.g., psychology, engineering, physical education, mathematics), at a number of educational establishments (e.g., high schools, vocational colleges, universities), in different levels of the educational paradigm (i.e., young learners, teenagers, and adults), and in a variety of academic contexts and linguistic backgrounds (e.g., Taiwan, Vietnam, and many European countries).

Interestingly, in contrast, some studies conducted on young learners in Asian countries found that CLIL instruction did not seem to exert a positive influence on students' content knowledge learning (e.g., Kang et al., 2010; Tsagkari, 2019). To elaborate, for example, Kang et al. (2010) were interested in assessing the influence of CLIL instruction on young Korean students in math and science classrooms, as contrasted to traditional teaching. To accomplish this objective, classes taught in English by native English speakers were compared to those taught in Korean by L1 Korean teachers. Based on the analysis and comparison of teacher talk produced, the CLIL-adopted Englishmedium content classes did not supply the learners with as much content knowledge as the L1-medium sessions. The L1-meidum lessons also inspired students to participate more actively in class. According to the researchers, young Korean learners and their relatively limited English proficiency appear to obstruct CLIL instruction's favorable influence.

To augment the CLIL scenario in a broader context of Asia, Tsagkari (2019) focused on the degree of applicability of CLIL implementation in the teaching contexts of Japan, Indonesia, and China. The 43 questionnaires completed by postgraduate students from the three countries were analyzed and found to be positive. The vast majority of respondents (28 out of 43) appeared enthusiastic with CLIL instruction. However, as language education stakeholders, they expressed worry and skepticism about crucial concerns relating to the instruction. They argued that in order to optimize the benefits of the instruction, materials, and resources should be developed, as well as teacher training programs to ensure that CLIL instruction is delivered properly.

In Thailand, the English language has been widely recognized as one of the vital tools to develop the country and thus received paramount attention. Consequently, a number of teaching approaches have been introduced principally to enhance Thai learners' English.Given the numerous studies as mentioned previously that have demonstrated the enormous benefits of CLIL, it is feasible that the Thai setting could be a forum for CLIL application. However, one encumbering factor that might inhibit successful CLIL application in the EFL context of Thailand needs to be explicitly mentioned. Thai learners' English proficiency is rather inadequate. In fact, the success of English education across the entire paradigm in Thailand is substandard, be it assessed by national or international examinations ( e. g., Education First, 2021; Kanoksilapatham, 2020; Kanoksilapatham \& Channuan, 2018). According to the world's largest test of English by Education First, taken by 2.2 million adults from 1112 countries and regions in 2021, Thailand was ranked 100. Of all 24 countries in Asia, Thailand was $22^{\text {th }}$ and classified as "very low proficiency". Since teachers have to be both content subject teachers and language teachers and as cautioned by Kang et al. (2010), CLIL implementation is likely to be too daunting in the elementary or high schools of Thailand for both students and teachers. That is, the students need to have a certain level of English proficiency to be able to cope with the instruction delivered by the content subject teachers in English. Meanwhile, the teachers must possess adequate English proficiency to deliver the CLIL instruction.

Taking these constraints into consideration, including the possibility of limited English proficiency among Thai school teachers and students as well as the implications generated from the findings of Kang et al. (2010) and Tsagkari (2019), this study highlights CLIL implementation in the EFL context of Thailand at university level because, potentially, higher education teachers are more likely to have a higher level of English expertise to handle and accommodate the demands of CLIL implementation. This study integrates CLIL instruction in teaching a research methodology course to enhance engineering students' content subject knowledge and English knowledge. The content subject knowledge refers to the ability to read research articles, whereas the English knowledge refers to the understanding of associated English vocabulary. The justification for focusing on the research methodology course is that, in order to graduate, these engineering students are required 
to conduct research and write a research report in English. Therefore, to help prepare the engineering students for this direction, CLIL instruction that enables them to be engaged in the discourse of research articles and improve their English seems appropriate. Seven weeks of CLIL implemented in this study were scaffolded by a number of supporting systems, including careful planning and close collaboration between the language and nonlanguage teachers, designing instructional materials and activities, and executing CLIL instruction in the classroom. This study adds to our understanding of CLIL application in Thailand.

\section{METHODS}

\section{Research Design}

The framework of research methods selected for this study is a mixed-method, incorporating both quantitative and qualitative data. Quantitative data were from multiple sources: two sets of pretests and posttests on research article reading and vocabulary skills, and a Likert scale questionnaire completed focusing on self-assessment of knowledge prior to and after CLIL instruction. Qualitative data were obtained from focus group discussion sessions to delve further into the effectiveness of CLIL implementation.

\section{Objectives \\ This research presents a study on CLIL instruction in a research methodology course offered to engineering students at a Thai university. Specifically, this current study aims to address three research questions: 1) What are the impacts of CLIL instruction on the students' research article reading skills? 2) What are the impacts of CLIL instruction on the students' vocabulary knowledge associated with the academic discourse of research articles? and 3) What are students' and content teachers' attitudes toward CLIL instruction?}

\section{Research setting}

This study was contextualized in the Department of Mechanical Engineering, Faculty of Engineering, of a medium-sized public university in the central region of Thailand. The classes offered by this department are usually conducted in Thai except for compulsory general English courses in the first year of study. However, given the fact that these general English courses are taught by Thai faculty members and that the engineering students' English competence might be somewhat limited, the Thai language could have been used on many occasions throughout the general English courses to facilitate learner comprehension.

\section{Participants}

According to the curriculum of the Department of Mechanical English, students are required to take general education courses during their first two years of study. During the last two years of their studies, they are required to take a number of mandatory courses, in addition to a few elective courses. For successful CLIL implementation, a course in which both English language teachers and content subject teachers can apply their expertise is required, which leaves only a few options for courses. Under careful examination, a research methodology course is the most idealistic of all available courses, (more detailed description of the course in the following section). At the time of the study, this course had a total number of 135 thirdyear undergraduate mechanical engineering students as participants. Due to the large number of students, to accommodate the students' class schedule, and to maximize the learning outcomes, two sections of the course were offered and scheduled on different days of the week. The students could choose the section according to their availability and convenience. Each section had a similar number of students (nearly 70 students each). Other factors relating to the participants, including their age, grade point average, and previous amount of English instruction received, were not referenced in this study. The participants of this study were treated as a single cohort after three years in the same study program.

\section{Research Methodology Course}

According to the curriculum, the undergraduate students in this mechanical engineering program are required to enrol in three consecutive courses focusing on research methodology. The first course of the series is offered in the second semester of each academic year - the time when this study was conducted. The course aims to develop learners' reading skills of mechanical engineering research articles in English. Thanks to this particular nature of the course and the way it could lend itself to the integration of the English language, this course was considered a clear fit for CLIL integration.

To better understand the classroom context of this study, a description of how the research methodology course was organized is imperative. The entire course of 15 class sessions (100 minutes per session) was taught by a team of five instructors with expertise in different areas of mechanical engineering, using a traditional face-to-face approach. All of the instructors have doctoral degrees and are actively involved in academic activities conducted in English, including oral presentations and academic writing.

The first half of the course, or the first eight class sessions, were independently taught by the five instructors, with an allocation of one to two class sessions each. As normally practiced, the Thai language was used as the medium of instruction. The purpose of the first half of the course is to expose the students to the research article genre, allowing them to be aware of the fundamentals of 
research methodology in mechanical engineering. The course also aims to highlight some of the commonly used current methodologies adopted in the field, justifications for selecting methodologies, and novel discoveries. Consequently, the content of the first half of the semester includes introducing the students to some general theoretical issues of research methodology in the discipline of mechanical engineering and exposing them to six research articles in English on a variety of topics that the instructors deemed appropriate for the students. The course was mostly lecture-based. Typically, the teachers assigned articles for the students to read. Then in class, the teachers went over the articles in detail. By the end of the first half of the course, the students were, to a certain extent, familiar with research article discourse.

\section{Materials and Instruments for CLIL Instruction}

In compliance with the objectives of the study, multiple instructional materials and assessment instruments were developed. Five sets of CLIL materials were developed for the next five class sessions. Assessment instruments were devised to estimate the students' content and vocabulary knowledge, as well as the students' and teachers' CLIL satisfaction. The following sections present in detail how these materials and instruments were developed.

\section{CLIL Instructional Materials}

The instructional materials employed for CLIL result from close collaboration between the language and non-language teachers. With the focus on research methodology course materials, multiple meetings between the engineering and English language teachers were held to discuss the scope and scale of the instructional materials in accordance with the course.

Because this research methodology course is the first one in the series, it is imperative that the engineering students are able to recognize the principal function of each research article section while reading. In this regard, Swales' (2004) genre analysis was relevant. In a nutshell, individual sections of research articles follow a typical organizational structure, consisting of moves and steps, which in turn can be recognized by a cluster of co-occurring linguistic features (for more details, see Kanoksilapatham, 2015). For example, the introduction section is to contextualize the study being presented, using a number of strategies, including topic centrality, topic generalization, and previous studies review. genre analysis provided the basis for designing and developing five sets of CLIL materials corresponding to the major sections of research articles in the following order of instruction: introduction, method, result, discussion, and abstract (or IMRD-Abstract).
As for CLIL material construction, to provide a smooth transition and to accommodate our research study, a number of arrangements were made and agreed upon by both the engineering instructors and the researchers. Initially, each engineering teacher was requested to nominate one research article on which to generate the CLIL instructional materials. To make sure that the five articles nominated were comparable and beneficial, they needed to be recent and comparable in length, containing the four explicit stand-alone headings of research article sections (i.e., IMRD). The five articles were then analyzed, using Swales' genre analysis. Based on Kanoksilapatham's framework (2015), the rhetorical structures pertaining to individual sections were identified. Each CLIL lesson pertaining to each of the five sections (IMRD and Abstract) consists of the organizational structure derived from genre analysis and some sentences corresponding to the moves and steps. A sample lesson on the method section is exemplified in Figure 1. The actual materials distributed and presented to the students were much more expansive.

The CLIL materials for the method section in Figure 1 encapsulate both the organizational structure derived from genre analysis and some sentences representing the section that could be used in the first 50 minutes of the CLIL session. To reinforce the meaningful and authentic use of the prominent linguistic features pertaining to the focused section (as presented at the bottom of Figure 1), in the remaining 50 minutes, the list of both grammatical and lexical features commonly used in the section was highlighted in the text and used to generate a variety of language activities (as shown in Figure 2). As depicted, some activities for the method lesson include past tense and passive voice (e.g., sentence completion, error recognition, and rearrangement) and move identification. In this last activity, a number of sentences belonging to different moves of the method section were presented, and the students as a group helped identify the moves to which they belong. It should be emphasized that all these activities were designed to stimulate student interaction and discussion among students.

To sum up, five sets of CLIL instructional materials were developed, focusing on the five sections of research articles (IMRD-Abstract). Each set consists of two major parts: the first part emphasizes how the content of each section is constructed, and the second part focuses on the linguistic features associated with the section. A variety of language activities were implemented in the second part of the lessons with the aim to reinforce the language features covered in the first half of the class sessions. 
Figure 1

CLIL Instructional Materials of the Method Section

\begin{tabular}{|c|c|c|}
\hline \multicolumn{3}{|c|}{ The method section } \\
\hline \multirow[t]{4}{*}{ Move 4: } & Descri & naterials/instruments/participants \\
\hline & Step 1: & Material list \\
\hline & Step 2: & Material source \\
\hline & Step 3: & Material background \\
\hline Move 5: & Detaili & cedures \\
\hline Move 6: & Describ & tistical procedures \\
\hline
\end{tabular}

Sample sentences corresponding to moves and steps

Move 4: Describing materials/instruments/participants

Step 1: Material list

- Three types of fuel namely diesel, natural gas and producer gas were used for the configurations modeled in this work.

Step 2: Material sources

- Biomass from perennial energy plants was obtained from field experiments $) 0.2$ ha for each plant( conducted at the University of Warmia and Mazury in Olsztyn )UWM(.

Step 3: Material backgrounds

- The bulk density of the materials used in briquette production ranged from $89.33 \mathrm{~kg} \mathrm{~m} \_3$ to $383.00 \mathrm{~kg} \mathrm{~m} \_3$ for chips of S. hemaphrodita and rapeseed oilcake.

Move 5: Detailing procedures

- In order to determine dimensions and unit mass, ten pellets were randomly selected in each experiment. The length $) \mathrm{L}($ and diameter $) \mathrm{D}$ ( of each pellet were measured using a digital vernier caliper. The mass of each pellet )m( was also weighed using a precision digital balance.

Move 6: Describing statistical procedures

- The analysis of data was done by constructing suitable tables and by using other statistical techniques such as mean, standard deviation, and t-test for mean scores. Percentage method was used to analyze Part-A of the questionnaire.

Grammatical features: passive, past tense

Lexical features - nouns: analysis, questionnaire, statistical techniques (mean, standard deviation, t-test, mean scores)

Lexical features - verbs: analyze, conduct, obtain, range, select, measure, use, weigh

Figure 2

Sample Activities of the Method Section

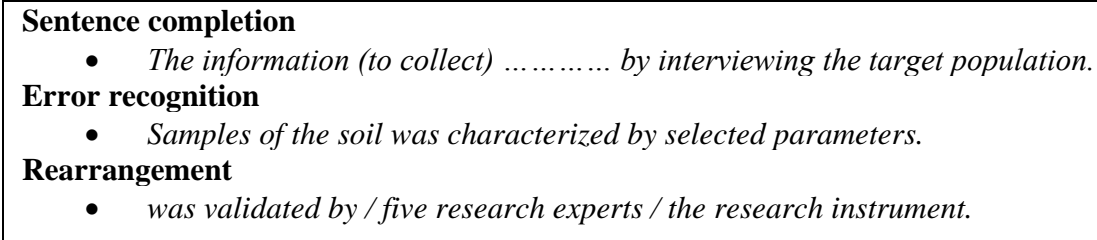

Move type identification task

Directions: Identify the moves used in the Method section. Write numbers 4, 5, or 6 in the space provided $(4=$ Move $4,5=$ Move 5, or $6=$ Move 6 ).

...... 1. The wind speech was measured in $3 \mathrm{hr}$ intervals at $10 \mathrm{~m}$ height of the earth's surface.

....... 2. The sampling frame was derived from the International Federation.

....... 3. The reliability of the items was examined using Cronbach alpha analysis.

\section{Assessment Instruments}

To assess the impact of CLIL instruction, a number of assessment tools were developed. To address the first two objectives of the study, a pretest and posttest design was employed. Two sets of identical pretests and posttests were constructed to assess the learners' English research article reading skills and associated English vocabulary knowledge (40 items each). Both the pretest and posttest on article reading skills consisted of 40 statements taken from the articles used in CLIL instruction. The students were required to identify the research article sections (IMRD( to which the statements are most likely to belong. All of the test items were validated by the five engineering instructors for accuracy and appropriateness. As shown in Figure 3, the answers for Statements 1 to 4 are $\mathrm{M}, \mathrm{R}, \mathrm{D}$, and I, respectively. 
Figure 3

Research Article Reading Test

Directions: Identify the section to which each statement belongs. Write the initial only (I, M, R or D) in the space provided for introduction, method, result, and discussion.

1. Questionnaires and survey forms were used as the research instruments.

....... 2. From the analysis, the biomass ratio on the water resistance had no effect.

3. There were several study limitations, including the lack of control of the characteristics of the substances.

4. Forest residues represent a major fuel source for potential bioenergy projects in many countries.

To examine the CLIL impact in enlarging the learners' vocabulary repertoire, 40 words taken from the first five subgroups of the Academic Word List (Coxhead, 2000) were used as the target language of the test to generate 40 statements. The students were to select one of the four options provided to complete each statement. Similar to the article reading skill test, these 40 items were validated by the five engineering instructors. Figure 4 displays five of the test items focusing on the words available, categorize, factor, similar, and analyze.

\section{Figure 4}

Vocabulary Test
Directions: Complete each blank with one of the four words provided.
1. Wind-surfers and kayaks are for rent at the beach.
a. available
b. individual
c. significant
d. economic
2. The students did an exercise in which they had to to its part of speech.
a. institute
b. transfer
c. categorize
d. regulate
3. Alcohol was a major in car accidents which claimed many lives last year.
a. formula
b. function
c. factor
d. finance
4. The planet Mars has a surface which is somewhat ............. to that of our moon.
a. specific
b. similar
c. legal
d. evident
5. Though it could take months to the data, the results will be useful to our project.
a. establish
b. constitute
c. analyze
d. legislate

To delve into the students' and teachers' CLIL instruction satisfaction, a questionnaire containing four 5-point Likert scale items was developed in Thai to elicit the students' self-assessment of perceived knowledge prior to and after CLIL instruction. The last three statements were added to elicit information regarding their satisfaction after CLIL instruction (Figure 5).

Figure 5

Questionnaire

Student questionnaire (4-item set for pre and post questionnaire, and 3 additional

items focusing on their satisfaction toward the end of CLIL instruction)

1. Your ability to read English research articles

2. Your knowledge of academic vocabulary

3. Your knowledge about the structure of individual research article sections

4. Your ability to read English research article abstracts

5. Your satisfaction of the CLIL instructional materials

6. Your satisfaction of the CLIL activities and tasks

7. Your general satisfaction of CLIL instruction

Finally, two separate sessions of focus group discussion were conducted to elicit reactions to the prompt: From your 7-class sessions of CLIL experience, what do you think of CLIL instruction?
One session was with ten randomly selected students, and the other with the five engineering instructors. 


\section{Procedures}

This section presents three major phases corresponding to the sequence of research activities: CLIL training, CLIL implementation, and data collection/analysis. Prior to the detailed description of these phases, certain arrangements were made before CLIL training.

CLIL requires close collaboration between the content teachers and the language teachers. In this study, multiple meetings and communications between the two parties commenced several weeks before CLIL implementation so that the engineering instructors had a clear understanding of this teaching approach. The collaboration led to the agreement that in the first half of the course, the engineering team would teach the way they usually did, focusing on six selected research articles and using Thai as the medium of instruction. The students were constantly informed of the CLIL instruction in the second half of the course conducted by the same instructors taking turns teaching, but in English. The five engineering teachers had no experience lecturing in English, but they were willing to participate in this CLIL project.

The engineering instructors and the researchers also discussed and agreed on the lesson plans of seven class sessions. Session 1 was reserved for CLIL overview and other housekeeping business including completion of the online consent form, pre-questionnaire, and two sets of pretests. The subsequent five sessions (Sessions 2 to 6) focused on individual article sections in the sequence of IMRD-Abstract. Session 7, the final session, was reserved for posttests administration, questionnaire completion, and student focus group discussion in Thai. The instructor focus group discussion was conducted outside class time in a meeting room.

\section{CLIL Training}

Five CLIL training sessions were organized by the researchers in English to facilitate subsequent CLIL implementation by the engineering instructors; each training session took place one week before the actual class and lasted 100 minutes. In the first half of each session, the CLIL lesson materials (a full version of an exemplified one in Figure 1) were distributed to the instructors. This first session also gave the instructors hands-on experience in analyzing articles texts, contributing substantially to a better understanding of the lessons. Based on the lesson files prepared by the researchers together with their training experience, the instructors responsible for their respective lessons prepared PowerPoint presentations for the students. Additional examples and modifications could be made as deemed necessary.

The second half of the training was reserved for language activities pertaining to the linguistic features predominantly found in each article section. A number of language activities were created to keep the students engaged, interactive, and focused, meanwhile reinforcing what was taught in the first part of the session as well as informally assessing whether the students understood how language functions in this particular academic discourse. All tasks were tried with the instructors so that they could thoroughly understand the purposes of the activities and implement them accordingly. Based on the instructors' constructive feedback and input, some activities were modified accordingly. The content teachers were informed that in the actual classroom, they had freedom to alter the task sequence or skip certain activities. To help the instructors with sizable class management, a research assistant with a master's degree in ELT was present in all CLIL sessions to help run and monitor the activities conducted in English.

\section{CLIL Implementation}

Several weeks before starting CLIL instruction, the instructors reminded the students that in the second half of the course, they would be participating in a teaching experiment with the content entirely in English. Here is a brief description of the activities taking place in each of the class sessions, all within the academic schedule, in a large classroom with plenty of space to move around for learning activities. The researchers were not present in the classroom to avoid potentially distracting the students and influencing the learning outcomes.

Session 1. CLIL was introduced to the students. Some changes that the students would experience include the switch to English as the language of instruction and the presence of the research assistant in the classroom to help with classroom activities. The students were assured that, despite these changes, this was an engineering research methodology class, not an English class. This notification was to highlight the importance of the course content, and to lessen the students' anxiety that might arise from English proficiency inadequacy. Then, a 4-item self-assessment questionnaire was completed individually online taking approximately five minutes. Subsequently, the two pretest sets on English article reading and vocabulary were distributed to the students, with a total of 60 minutes allocated. Finally, five articles selected by the instructors were distributed as a reading package for CLIL instruction in Sessions 2, $3,4,5$, and 6 .

Session 2. CLIL implementation was launched by one of the instructors focusing on the introduction section. To keep a balance between the focus on content and language, the five engineering instructors were encouraged to spend the first 50 minutes on the instruction of content, and the remaining 50 minutes on language-related activities supported by the assistant.

Sessions 3, 4, 5, and 6. During the four successive weeks, a series of four CLIL instruction 
sessions was conducted, focusing on the sections of MRD-Abstract. These four sessions proceeded in a similar manner to that in Session 2.

Session 7. In this final CLIL implementation class, the assessment activities conducted in Session 1 were repeated with minimal change. That is, a 7item self-assessment questionnaire was administered online, with three additional questions to elicit the students' opinions regarding their CLIL experience satisfaction. The two posttests, identical to the pretests, on English article reading and vocabulary were administered in the classroom. Finally, to delve further into the effectiveness of CLIL implementation, 10 randomly selected students were requested to participate in a 10-minute focus group discussion. A few hours later on the same day, a similar 10-minute focus group discussion with the instructors was conducted. From these focus group discussion sessions, personal opinions could be creatively and constructively expressed.

\section{Data Collection and Analysis}

To examine the impact of the CLIL implementation on the students, the two sets of pretest and posttest scores of research article reading and English vocabulary were analyzed by descriptive statistics. Subsequently, to observe the CLIL impact on knowledge gain, a t-test was conducted on the pretest and posttest mean scores. Students who were not able to complete all the tests were excluded from the analysis. To examine whether the students were satisfied with CLIL instruction, the data from the additional three items in the questionnaire administered in Session 7 were collected and quantitatively analyzed. The data collected from the two sessions of focus group discussion were coded based on the themes that emerged.

\section{FINDINGS}

The final pool of participants in this study consists of 135 mechanical engineering students enrolled in the research methodology course. The analysis of the pretest and posttest scores pertaining to their reading skills (i.e., identifying the research article sections to which individual statements belong) is depicted in Table 1 .

Table 1

Pretest and Posttest Scores of the Participants' Reading Skills $(N=40)$

\begin{tabular}{|c|c|c|c|c|c|c|c|}
\hline & Max & Min & Mean & $S D$ & $t$ & $d f$ & $p$ \\
\hline Pretest & 31.00 & 7.00 & 18.3852 & 4.60691 & \multirow{2}{*}{-3.024} & \multirow{2}{*}{134} & \multirow{2}{*}{$.003 *$} \\
\hline Posttest & 36.00 & 7.00 & 19.7556 & 6.22161 & & & \\
\hline
\end{tabular}

As shown, from the research article reading test, some of the participants demonstrated quite satisfactory performance, reaching the highest scores of 31 and 36 from a total score of 40 points in the pretest and posttest, respectively. However, in scrutiny, the relatively low mean pretest score of 18.38 indicated that some participants had difficulty with this test. The posttest score analysis, after CLIL instruction, showed that the mean score was slightly higher at 19.76. The subsequent t-test analysis conducted demonstrates that the knowledge gain was significant at 0.003 . In short, this study shows that CLIL instruction did exert a positive impact in sharpening the participants' skills of reading engineering research articles.

Now let us consider the scores of the English vocabulary tests associated with the research articles, the second objective of this study. The analysis of the pretest and posttest scores of this part is shown in Table 2.

Table 2

Pretest and Posttest scores of the Participants' Vocabulary Knowledge $(N=40)$

\begin{tabular}{lccccccc}
\hline & Max & Min & Mean & SD & t & df & $\boldsymbol{p}$ \\
\hline Pretest & 29.00 & 4.00 & 15.8222 & 5.93983 & \multirow{2}{*}{-2.809} & \multirow{2}{*}{134} & $.006 *$ \\
Posttest & 32.00 & 6.00 & 16.7407 & 5.52331 & & &
\end{tabular}

The participants did not seem to perform well in this vocabulary test either. As shown, the mean score was quite low (15.8) in the pretest but rose slightly in the posttest (16.7). The minimum scores of the pretest and posttest were also rather low. The t-test analysis of the mean scores showed that the gain score was significantly different, although not as substantial as that of the reading skills.

The last set of quantitative data addresses the third objective of the study. That is, it aims to examine the students' self-assessment and attitudes toward CLIL instruction. In this regard, the analysis of the 4-item questionnaire prior to CLIL exposure and the 7-item questionnaire post exposure is presented in Table 3. The cutoff mean scores and their associated interpretations are as follows: 1.001.80 (very low), 1.81-2.60 (low), 2.61-3.40 (moderate), 3.41-4.20 (high), and 4.21-5.00 (very high). 
Table 3

Comparison of the Participants' Self-Assessment and Attitudes

\begin{tabular}{lll}
\hline \multicolumn{1}{c}{ Statement } & $\begin{array}{l}\text { Pretest } \\
\text { Mean Score }\end{array}$ & $\begin{array}{l}\text { Posttest } \\
\text { Mean Score }\end{array}$ \\
\hline 1. Your ability to read English research articles & 2.34 & 3.24 \\
& Low & $\begin{array}{l}\text { Moderate } \\
3.18\end{array}$ \\
2. Your knowledge of academic vocabulary & 2.22 & Moderate \\
& Low & 3.27 \\
3. Your knowledge about the structure of individual research article sections & 2.29 & 3.25 \\
Low & Moderate \\
4. Your ability to read English research article abstracts & 2.30 & 3.62 \\
5. Your satisfaction of the CLIL instructional materials & Low & High \\
& N/A & 3.83 \\
6. Your satisfaction of the CLIL activities and tasks & N/A & High \\
8. Your general satisfaction of CLIL instruction & & 3.74 \\
& N/A & High \\
\hline
\end{tabular}

Based on their self-assessment, it was evident that the participants believed they possessed a low level of relative knowledge and ability prior to CLIL instruction, with a narrow range from 2.22 to 2.34 . However, after CLIL instruction, the participants assessed themselves as moderately proficient in all areas, with the mean scores ranging from 3.18 to 3.25. The findings substantiate the t-test analyses depicted in Tables 1 and 2, indicating that they increased their knowledge in those areas. As for the last three items in Table 3, the participants were highly satisfied with CLIL instruction, particularly with regard to the activities (3.83). The data thus revealed their positive attitudes toward CLIL instruction, yielding a general satisfaction rate of 3.74. Furthermore, the qualitative analysis of the data pertaining to the two focus group discussion sessions with the students and the instructors is quite interesting. To reiterate, the discussants were to react to the prompt: "From your 7-class sessions of CLIL experience, what do you think of CLIL instruction?" Based on the student group discussion, three emerging themes were coded: the CLIL approach, language activities, and future expectations.

First, as far as CLIL instruction is concerned, most student discussants expressed their content and positive impression with this instruction because they could learn about current research methodology in mechanical engineering and improve the English language at the same time. The knowledge gained from this CLIL integration enabled them to learn a wide variety of vocabulary items and understand the function of linguistic features, facilitating the task of reading research articles. To elaborate, prior to CLIL instruction, they were unaware that each section of research articles tended to follow a particular organizational structure, signifying key material to be included. For example, an abstract, notwithstanding its length, comprises a mandatory element of "result reporting." Moreover, they learned that past tense and passive voice, rather than active voice, are prevalent linguistic features in result statements in their academic area as a result of this CLIL instruction.

With this knowledge, they became more competent in reading abstracts. All of them congruently agreed that they had no prior experience with CLIL instruction, but it made the class much more interesting. Most of them were pleasantly surprised to find that the English language could be interestingly harnessed and integrated into an engineering course.

Language activities are another domain that the discussants mentioned positively at great length, in congruence with the questionnaire results. One of them was so engaged in the activities that he hardly stayed still in the second half of the class sessions. Another student admitted that group work and peer collaboration to complete language activities was a rewarding experience. It made her realize the value of teamwork skills and the opportunities to exchange opinions among her peers. In addition to the enjoyment, she was able to understand and appreciate the lessons better. One discussant mentioned a specific task of sequencing sentences to form a well-written abstract. He became aware of the crucial role of organization and the choice of linguistic features that serve communicative functions in the academic discourse. Many of them acknowledged that the activities kept them alert and engaged throughout the sessions, allowing them to retain what was learned. While many found the activities educational and entertaining, two discussants found the language activities somewhat challenging. They wished they had more time to work on certain language activities due to their limited English competency. To exemplify, the two pieces of feedback obtained from the student focus group discussion were originally in spoken Thai, and their corresponding verbatim translation is as follows.

a. I had no idea that this research methodology class would be so informative and entertaining. Due to the nature of the language tasks, we 
were required to run around the large classroom looking for missing pieces of information. There isn't enough time for me to check incoming LINE text messages. That's fantastic!

b. I enjoyed the language activity in which we, as a team, had to deconstruct an authentic abstract into smaller chunks based on various communicative functions. Another activity I liked was sequencing the sentences provided in order to form a well-written abstract. Well, there was a lot of wrangling and arguing among our team members. These activities came in handy when I was writing an English abstract.

The third theme that emerged from the focus group discussion is about future expectations. Many of them asked the instructors whether CLIL instruction would be applied to other engineering courses. They felt the CLIL approach would be very practical and useful for working on their research project in their final year of study. Many discussants implored that they would like the chance to participate in CLIL instruction again in the future.

The instructor focus group discussion took place in a small meeting room about two hours after the class. In response to the prompt, they agreed entirely that CLIL instruction is extremely beneficial, particularly for themselves because they had a better understanding of how research articles were constructed and a better grasp of how the English language works in this academic genre. All of them witnessed that the students were motivated and enthusiastic to learn and participate in classroom activities, something that the Department had tried over the years, unfortunately with little success. All of them admitted that this is the first time they had to deliver their teaching in English, and it was not easy. Without CLIL training and support, delivering a 100-minute CLIL session would have been too challenging. However, they agreed that this CLIL experience was valuable because not only were the students learning, but "we" teachers were learning too. Specifically, they did not know that each article section is governed by a structural pattern so learning about this was intriguing and exciting. To illustrate, here are two excerpts from the content teachers' feedback originally delivered in spoken Thai.

a. I believe this teaching approach allows the students to apply what they have learned when faced with the need to read and write research articles in English. I wish that this teaching approach could be used in other mechanical engineering courses.

b. I can see positive changes occurring in this course. The students were ecstatic to be learning. My engineering students were becoming more interested in learning English. That's a good sign!

With reference to language activities, they were amazed to learn that a research methodology course could be manipulated in such a way to keep the students actively engaged and alert at all times. Clearly, the students were strongly motivated when participating in these activities, contributing substantially to the class achievement. However, despite the benefits of the activities, they wonder how they could manage this endeavor without the researchers' preparation and materials. This challenge results from not only their limited expertise and knowledge in teaching pedagogy but also the tremendous amount of time needed in designing and preparing these activities.

The final remark to be reported here is about the challenge to conduct classes in English. Even though the teachers were somewhat proficient in English, they realized that teaching using English as the medium of instruction for a period of 100 minutes could be quite demanding. Thus, they were very anxious at the beginning of CLIL instruction. They stated that this would not have been possible or as efficient without the series of training sessions offered. They learned not only how to teach, but also how to craft or improvise "teacher talk" in English. Even though they were less anxious as time went by, they were sure that the students could pick up on their anxiety and appreciated their efforts. To exemplify, one of the teachers admitted that he was so focused on delivering the course content in English that he neglected to include teacher talk like "Now, let's move on to the next topic." or "Let's take a look at this figure." However, from the training sessions, he discovered that integrating teacher talk helped him relax and meanwhile allowed him time to breathe. It also gave the students more time to prepare for the activities that follow. In a sense, from this experience, the instructors could be seen as role models for their students in that no one should stop learning English, no matter what their discipline is. Finally, all of the instructors wish to adopt CLIL instruction in their future courses, either for the entire course or at least for an extended period of time. Evidently, substantial support or scaffolding from language teachers is indispensable in order to professionally deliver in a CLIL class.

\section{DISCUSSION}

This article presents a study of teaching research methodology content in English to university students using CLIL instruction. It aims to examine whether CLIL in an EFL context could promote the learners' content subject knowledge, enlarge associated English vocabulary, and instill positive learning attitudes. Based on the quantitative results 
of the pretest and posttest scores, the learners demonstrated an enhanced ability in understanding the structure of research articles and vocabulary associated with the research article discourse. The impact of CLIL instruction on vocabulary was in congruence with previous studies of Heras and Lasagabaster (2015) and Crossman (2018), both of which reported vocabulary growth in high school and university students, respectively. A positive impact also manifested in the students' satisfaction of CLIL instruction reflected in their questionnaires and focus group discussion sessions.

At this juncture, the findings of this study substantiate Tsagkari's (2019) observation that instructional materials play a vital role determining CLIL integration. As demonstrated in this study, instructional materials including language tasks and activities were meticulously devised, trialled, and finally executed by the language teachers. We were concerned that delegating language tasks and activities to content teachers would be too daunting. Therefore, in accordance with Tsagkari's warning, CLIL teacher training sessions implemented in this project are critical to ensuring successful CLIL instruction. This study's accomplishment with CLIL instruction also lends support to Kang et al.'s (2010) caution that, in order to optimize the benefits of CLIL, learners' levels of English proficiency must be considered. CLIL implementation success, especially in the context of English as a foreign language like Thailand, is partly due to the fact that these university students possess a certain level of English proficiency that can be pushed further. CLIL integration delivered to younger students may seriously jeopardize favorable outcomes.

Pedagogical implications from this study are multiple. This study indicates that CLIL represents an effective approach that both language and nonlanguage teachers can collaborate to fruitfully focus on both the content subject and the language. As demonstrated by the findings of the study, the students gained additional content subject knowledge and expanded their English vocabulary repertoire. The positive impacts of CLIL, in fact, are not only on the learners but also the teachers involved. To elaborate, although content subject teachers are experts and professionals in their disciplines, they might feel somewhat reluctant to take on the responsibility of language teaching (Hurajova, 2021). Specifically, Kao ) 2020) maintains that CLIL instructors might lack confidence, as is the case in this current study, as they have an insufficient level of language skills to implement CLIL. However, with additional CLIL exposure and support, the instructors of this study became more competent in delivering CLIL lessons in English. In other words, CLIL provides a channel for non-language teachers to develop their English competence and boost their confidence in using
English as a medium of instruction and for classroom interaction.

Once embarking upon CLIL instruction, content subject instructors are compelled to be not only knowledgeable of English. In congruence with Pérez Cañado (2016), they become aware of pedagogical strategies and techniques that work. The content subject teachers are likely to feel awkward when planning and delivering CLIL lessons in English, as revealed in this study. However, all instructors in the present study asserted that language activities designed and planned in CLIL instruction play an integral and indispensable role in empowering students. Therefore, to alleviate the instructors' concerns, in this study, language activities designed based on teaching pedagogy and strategies were devised for the instructors as a scaffolding device to reinforce what the learners had previously acquired. Moreover, the engineering instructors observed that the students reacted positively, enthusiastically, and energetically to the language tasks and activities. This observation highlights the role of systematic integration of content and language in determining CLIL success. In this regard, the engineering instructors became aware of language teaching pedagogy and its impact in turning a regular class into a more interactive and lively class.

All of the benefits mentioned above would not have been possible without multiple CLIL training sessions before CLIL implementation. It is generally assumed that teachers of all disciplines need to constantly participate in professional development by attending workshops and conferences. However, this assumption might need to be revised. In this study, the engineering instructors actively participated in professional development right in their own classrooms. That is, the language and the engineering instructors worked collaboratively from the very beginning to make sure that the engineering instructors clearly understood the CLIL principles, and that CLIL implementation was properly conducted. It is anticipated that the collaboration between the non-language instructors and the language teachers in the stages of syllabus design, material development, test construction, and activity execution will empower the engineering instructors to thrive and maximize the learners' learning outcomes.

CLIL seems to be tremendously effective when applied in many European countries (e.g., Fazzi \& Lasagabaster, 2021; Mahan, 2020; Pérez Cañado, 2020). However, in EFL contexts, the impact of CLIL does not seem to be that clear. As depicted by multiple studies on young learners (e.g., Kang et al., 2010; Tsagkari, 2019), EFL learners' limited English proficiency was speculated to be a hindering factor. As shown in this study, CLIL was successful when conducted at a university in Thailand. Evidently, university learners' English proficiency 
can vary, especially in a large heterogenous class. However, in general, these students have had substantial English exposure and developed their English proficiency to a certain extent. In addition, as stated in their focus group discussion, they possess the motivation and understanding that English is essential for not only their academic endeavors, but also future undertakings. However, it needs to be pointed out that the English proficiency of CLIL teachers plays a vital part too, contributing to the successful delivery of the content and provision of comprehensible language input to learners. Although they initially lacked confidence in delivering classes in English, they were determined to complete CLIL instruction in English. In short, as supported by this study, CLIL success is not determined by the students' English proficiency alone, but also that of the instructors.

Finally, this study represents only one successful model of CLIL implementation at a university in Thailand. This particular model might not work at other academic establishments. Therefore, CLIL implementation still offers plenty of room for flexibility and tailoring to accommodate individual contexts. Furthermore, in this modern global society, tremendous needs and demands have called for collaborative and engaged pedagogy; therefore, it is hoped that this study can add to the developing body of knowledge about different forms of CLIL programs for different levels, learning contexts, learner groups, and academic disciplines.

\section{CONCLUSION}

This article reports the fruitful collaboration between language teachers and engineering teachers in a CLIL class at a public university. This study provides crucial insights into CLIL implementation in an EFL context of Thailand. Through CLIL instruction, the students' ability in reading engineering research articles and associated vocabulary knowledge improved. CLIL benefits also extend to the content subject teachers, rendering them more competent and confident in English, while being engaged in teaching a content subject course. The findings of this study raise a number of issues that could lead to a more rigorous CLIL implementation. For example, large-scale research to examine the non-language teachers' challenges in teaching content subjects using CLIL will contribute to a better understanding of how CLIL works. It would be interesting to observe CLIL integration and its effects in longitudinal studies focusing on productive skills like speaking and writing. Another research area that deserves further scrutiny is whether students' English proficiency is associated with their success in, or satisfaction with, CLIL instruction. Given the limited scale and scope of this study, a broader study integrating a number of academic disciplines and a full course of CLIL instruction will definitely allow us to accurately validate the positive outcomes of CLIL instruction. Additionally, classroom observation needs to be taken into consideration when examining the extent that the non-language teachers use English in CLIL so that the language teachers can address these issues for further collaboration. Finally, for advanced CLIL classes, tests executed can be more challenging, such as requiring the learners to write engineering research article sections in English, using the appropriate organizational structure and linguistic features.

\section{ACKNOWLEDGEMENT}

This work was supported by Thailand Research Fund under Grant No. RDG610030.

\section{REFERENCES}

Coyle, D., Hood, P., \& Marsh, D. )2010(. CLIL: Content and language integrated learning. Cambridge University Press. https://doi.org/10.1016/j.system.2011.01.001

Coxhead, A. )2000(. Selecting vocabulary: Academic word list. UEFAP. http://www.uefap.com/vocab/select/awl.htm

Crossman, K. (2018). Immersed in academic English: Vocabulary and academic outcomes of a CLIL university preparation course. International Journal of Bilingual Education and Bilingualism, 21(5), 564-577. https://doi.org/10.1080/13670050.2018.149469 8

Education First. (2021). The world's largest ranking of countries and regions by English skills. Special report. https://www.ef.co.th/epi/

Fan, C. C., \& Lo, Y. Y. )2016(. Interdisciplinary collaboration to promote L2 Science literacy in Hong Kong. In A. Tajino, T. Stewart, \& D. Dalsky )Eds.(, Team teaching and team learning in the Language classroom: Collaboration for innovation in ELT )pp. 94112(. Routledge.

Fazzi, F., \& Lasagabaster, D. (2021). Learning beyond the classroom: students' attitudes towards the integration of CLIL and museumbased pedagogies. Innovation in Language Learning and Teaching, 15(2), 156-168. https://doi.org/10.1080/17501229.2020.171463 0

Heras, A., \& Lasagabaster, D. (2015). The impact of CLIL on affective factors and vocabulary learning. Language Teaching Research, 19(1), 70-88. https://doi.org/10.1177/1362168814541736

Hurajova, L. (2021). Can close cooperation between ESP/CLIL experts and disciplinary teachers in higher education lead to fostering English 
education environment. Journal of Teaching English for Specific and Academic Purposes, 9, 129-136. https://doi.org/10.22190/JTESAP2101129H

Kalay, D. (2021). Receptive vocabulary knowledge of university students in CLIL instruction, European Journal of Foreign Language Testing, 5(3), 33-52. https://doi.org/10.46827/ejfl.v5i3.3527

Kang, Y., Hwang, H., Nam, K., \& Choi, Y. (2010). Comparison of teacher talk of Korean and native English-speaking teachers: On the efficiency of delivering content knowledge in EFL contexts, Journal of Asia TEFL, 7(2), 128. https://www.earticle.net/Article/A182276

Kanoksilapatham, B. (2015). Distinguishing textual features characterizing structural variation in research articles across three engineering subdiscipline corpora. English for Specific Purposes, 37, 74-86. https://doi.org/10.1016/j.esp.2014.06.008

Kanoksilapatham, B., \& Channuan, P. (2018). EFL learners' and teachers' positive attitudes towards local community based instruction. Indonesian Journal of Applied Linguistics, 7(3), 504-514. https://doi.org/10.17509/ijal.v7i3.9790

Kanoksilapatham, B. (2020). Local culture preservation through southern Thainess-based English lessons. KEMANUSIAAN: The Asian Journal of Humanities, 27(2). https://doi.org/10.21315/kajh2020.27.2.10

Kao, Y. T. (2020). Understanding and addressing the challenges of teaching an online CLIL course: a teacher education study. International Journal of Bilingual Education and Bilingualism, 1-20. https://doi.org/10.1080/13670050.2020.171372 3

Mahan, K. R. (2020). The comprehending teacher: Scaffolding in content and language integrated learning (CLIL). The Language Learning Journal, 1-15. https://doi.org/10.1080/09571736.2019.170587 9

Marsh, D. )2000(. CLIL: An interview with Professor David Marsh. Journal of Education and Development, 29. http://ihjournal.com/content-and-languageintegrated-learning

Navarro-Guzman, J., Romero-Alfaro, E., MenachoJimenez, I., \& Aragon-Mendizabal, E. (2021). Teaching psychology at university using the content and language integrated learning (CLIL) approach, Porta Linguarum, 35, 77-91. https://doi.org/10.30827/portalin.v0i35.16858

Pérez Cañado, M. L. )2016(. Are teachers ready for CLIL? Evidence from a European study," European Journal of Teacher Education, 39, 202-221. https://doi.org/10.1080/02619768.2016.113810 4

Pérez Cañado, M. L. (2020). CLIL and elitism: Myth or reality? The Language Learning Journal, 48(1), 4-17. https://doi.org/10.1080/09571736.2019.164587 2

Swales, J. )2004(. Research genres: Explorations and applications. Cambridge University Press.

Thuy, T. \& Nguyen, L. (2016). Reconsidering the first steps of CLIL implementation in Vietnam. European Journal of Language Policy, 8(1), 29-57. https://doi.org/10.3828/EJLP.2016.4

Tsagkari, A. (2019). Students' Perceptions on CLIL Implementation in China, Japan and Indonesia. 鹿児島純心女子短期大学研究紀 要, (49), 31-58.

Yang, W. (2015). Content and language integrated learning next in Asia: Evidence of learners' achievement in CLIL education from a Taiwan tertiary degree programme. International Journal of Bilingual Education and Bilingualism, 18(4), 361-382. https://doi.org/10.1080/13670050.2014.904840 